\title{
Long-chain $n-3$ polyunsaturated fatty acids and blood pressure
}

\author{
Yuichiro Yano \\ Hypertension Research (2012) 35, 967-968; doi:10.1038/hr.2012.102; published online 28 June 2012
}

A large body of evidence from experimental, clinical and epidemiological research has demonstrated the potential benefits of long-chain $n-3$ polyunsaturated fatty acids (PUFAs), eicosapentaenoic acid (EPA) and docosahexaenoic acid (DHA) for cardiovascular health, such as anti-atherogenic effects, plaque stability effects, improvement of endothelial or platelet function and anti-arrhythmic effects. ${ }^{1}$ These beneficial effects of $n-3$ PUFA are derived from a myriad of molecular pathways, including alteration of the physicochemical properties of cell and organelle-membrane structure and function, direct interaction with and modulation of membrane channels and proteins, regulation of gene expression through nuclear receptors and transcription factors, changes in arachidonic acid-derived eicosanoid profiles and conversion of $n-3$ PUFA to bioactive metabolites. ${ }^{1}$

One of the recent topics in the field of $n-3$ PUFA research is its effects on blood pressure (BP) levels. Although the results of the different studies have not been consistent, several observational studies have demonstrated an association of $n-3$ PUFA with low BP levels and interventional studies using n-3 PUFA supplementation have shown its BP-lowering effects. The population-based International Study of Macro- and Micronutrients and Blood Pressure surveyed 4680 men and women aged 40-59 from 17 population samples and found an inverse association between BP and $n$-3 PUFA intake from foods while controlling for multiple possible confounders, although the estimated size of the effect was small, that is, $<1.0 \mathrm{~mm} \mathrm{Hg}$

Dr Y Yano is at the Department of Medicine, Division of Cardiovascular Medicine, Jichi Medical University School of Medicine, Tochigi, Japan.

E-mail:yyano@jichi.jp with $1.9 \mathrm{~g}$ per day higher $n$-3 PUFA intake (about $1.9 \mathrm{~g}$ per day). ${ }^{2}$ In interventional studies, Appel et al. ${ }^{3}$ estimated that BP was reduced by $-1.0 /-0.5 \mathrm{~mm} \mathrm{Hg}$ in normotensive subjects and by $-5.5 /-3.5 \mathrm{~mm} \mathrm{Hg}$ in untreated hypertensive subjects for an average supplementation with $>3 \mathrm{~g}$ per day of $n$-3 PUFA, suggesting that the BP-lowering effect of $n$-3 PUFA is stronger in hypertensive subjects. In addition, a meta-analysis showed that the anti-hypertensive effects of $n$-3 PUFA were dose-dependent $(-0.66 /$ $-0.35 \mathrm{~mm} \mathrm{Hg} \mathrm{g}^{-1} n-3$ PUFA) and the effects tended to be larger in middle-aged and older persons $(\geqslant 45$ years $)$ or those with excess clinical atherosclerotic burden. ${ }^{4,5}$ The postulated mechanisms of the hypotensive effects of n-3 PUFA are summarized in Figure 1.

In the current issue of Hypertension Research, Virtanen et al. ${ }^{6}$ strengthen the association between $n-3$ PUFA and BP by using a cross-sectional analysis $(n=768)$ that includes middle-aged and older subjects, and those who did not have a previous history of ischemic heart disease, stroke, diabetes or hypertension treatment. They showed that circulatory $n$-3 PUFA levels were inversely associated with systolic BP and pulse pressure but not with diastolic BP. As the present study was conducted at relatively high age (mean age $\sim \leqslant 60$ years), the clinical implication of diastolic BP seems to have less impact than systolic BP. In this study, BP was measured by the mean of six BP values in an office setting and the serum-esterified and non-esterified fatty acids were determined by one gas chromatographic run (not determined by dietary intake). The assessments would thus seem to be reasonably accurate.

Circulating n-3 PUFA levels are derived mainly from fish intake, which also provides various vitamins, minerals and elements,

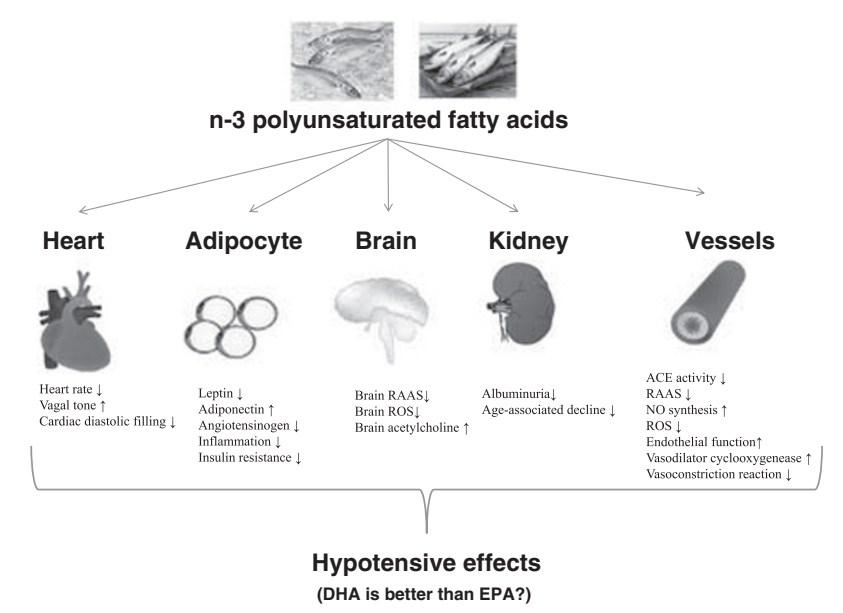

Figure 1 Possible mechanisms of the BP-lowering effects of $n-3$ polyunsaturated fatty acids. These mechanisms were derived from experimental and clinical studies. ACE, angiotensin-converting enzyme; RAAS, renin-angiotensin-aldosterone system; NO, nitric oxide; ROS, reactive oxygen species. A full color version of this figure is available at the Hypertension Research journal online. 
such as vitamin D and selenium, as well as vital proteins. In addition, fish can serve as a dietary replacement for less-healthy protein sources, such as red meat, and the reverse is also true as subjects who are unwilling to eat fish or related products may show unfavorable dietary habits. ${ }^{1}$ These are important confounding factors in the association between $n$-3 PUFA and BP. In fact, in the present study, ${ }^{6}$ subjects with high circulating n-3 PUFA levels were likely to have a higher income, education, urinary potassium excretion and vegetable (excluding potatoes) intake, and a lower intake of energy and meat and meat products, and were less likely to smoke. Even though the authors adjusted for such confounding factors, residual confounding due to unknown or incompletely measured characteristics could partly explain the present findings.

In contrast to the beneficial effects of $n-3$ PUFA, concerns have been raised about the potential harm from contaminants in some fish, such as methylmercury, dioxins and polychlorinated biphenyls. ${ }^{1}$ Epidemiological evidence suggests a negative impact of methylmercury on the cardiovascular system, but the effects on $\mathrm{BP}$ are inconsistent. ${ }^{7,8} \mathrm{~A}$ recent survey of Inuit from Nunavik, who are highly exposed to environmental methylmercury, showed that circulating methylmercury levels were associated with increasing systolic BP and pulse pressure after controlling for the confounding factors. ${ }^{7}$ Intriguingly, when this association was adjusted by $n$ - 3 PUFA and selenium, the association between methylmercury and BP became more prominent. In the present analysis, ${ }^{6}$ the methylmercury level in hair was not associated with BP and it did not modify the association between $n$-3 PUFA and BP. The discrepant results may be partly derived from the differences in culture among the study populations; for example, BP levels of non-fish consumers are likely to be influenced by methylmercury intake. ${ }^{7,8}$
In most fish species methylmercury levels are quite low, and thus the health benefits of consuming an appropriate amount of fish can outweigh such potential risks.

Lastly, we would like to comment on another important aspect of n-3 PUFA. The high-order collinearity of EPA and DHA intake from foods limits the ability to determine how each of these factors influences cardiovascular protection. However, data now available demonstrate that DHA and EPA exert slightly different hemodynamic and anti-atherogenic effects. ${ }^{1,9,10}$ In clinical studies, supplementation with DHA leads to more favorable effects on forearm vascular reactivity and 24-h BP and pulse rate levels in hyperlipidemic obese patients compared to supplementation with EPA.9,10 These results may be partly explained by the differential influence on endothelial nitric oxide production between EPA and DHA. ${ }^{11}$ Moreover, some large prospective studies have demonstrated that higher circulating DHA levels, but not higher EPA levels, were inversely associated with the incidence of heart failure or atrial fibrillation, and both these pathophysiologies can be attributed in part to high BP burden. ${ }^{12,13}$ In the present study, ${ }^{6}$ Virtanen et al., examined the association of BP levels with EPA, DHA or docosapentaenoic acid. They found that each of the associations were similar. Differences in study designs, population characteristics and types and dosage of $n-3$ PUFA are possible explanations for the discrepancy between their results and those of the aforementioned studies. It is of both theoretical interest and practical importance to understand the relative hypotensive capacity of EPA and DHA, and which compounds we should choose as a supplement or for drug intervention (for example, is only EPA or DHA sufficient, or are both needed?) to achieve more cardiovascular protection. Confirmation of these unresolved issues by interventional studies with appropriate numbers of participants will be needed in the future.

1 Mozaffarian D, Wu JH. Omega-3 fatty acids and cardiovascular disease: effects on risk factors, molecular pathways, and clinical events. J Am Coll Cardiol 2011; 58: 2047-2067.

2 Ueshima H, Stamler J, Elliott P, Chan Q, Brown IJ, Carnethon MR, Daviglus ML, He K, Moag-Stahlberg A Rodriguez BL, Steffen LM, Van Horn L, Yarnell J, Zhou B. INTERMAP Research Group. Food omega-3 fatty acid intake of individuals (total, linolenic acid, long-chain) and their blood pressure: INTERMAP study. Hyperten sion 2007; 50: 313-319.

3 Appel LJ, Miller 3rd ER, Seidler AJ, Whelton PK. Does supplementation of diet with 'fish oil' reduce blood pressure? A meta-analysis of controlled clinical trials. Arch Intern Med 1993; 153: 1429-1438.

4 Geleijnse JM, Giltay EJ, Grobbee DE, Donders AR, Kok FJ. Blood pressure response to fish oil supplementation: metaregression analysis of randomized trials. J Hypertens 2002; 20: 1493-1499.

5 Morris MC, Sacks F, Rosner B. Does fish oil lower blood pressure? A meta-analysis of controlled trials. Circulation 1993; 88: 523-533.

6 Virtanen JK, Nyantika AN, Kauhanen J, Voutilainen S, Tuomainen T-P. Serum long-chain n-3 polyunsaturated fatty acids, methylmercury and blood pressure in an older population. Hypertens Res 2012; 35: 1000-1004.

7 Valera B, Dewailly E, Poirier P. Environmental mercury exposure and blood pressure among Nunavik Inuit adults. Hypertension 2009; 54: 981-986.

8 Vupputuri S, Longnecker MP, Daniels JL, Guo X, Sandler DP. Blood mercury level and blood pressure among US women: results from the National Health and Nutrition Examination Survey 1999-2000. Environ Res 2005; 97: 195-200.

9 Mori TA, Bao DQ, Burke V, Puddey IB, Beilin LJ. Docosahexaenoic acid but not eicosapentaenoic acid lowers ambulatory blood pressure and heart rate in humans. Hypertension 1999; 34: 253-260.

10 Mori TA, Watts GF, Burke V, Hilme E, Puddey IB Beilin LJ. Differential effects of eicosapentaenoic acid and docosahexaenoic acid on vascular reactivity of the forearm microcirculation in hyperlipidemic, overweight men. Circulation 2000; 102: 1264-1249.

11 Abeywardena MY, Head RJ. Longchain n-3 polyunsaturated fatty acids and blood vessel function. Cardiovasc Res 2001; 52: 361-371.

12 Yamagishi K, Nettleton JA, Folsom ARARIC Study Investigators. Plasma fatty acid composition and incident heart failure in middle-aged adults: the Atherosclerosis Risk in Communities (ARIC) Study. Am Heart J 2008; 156: 965-974.

13 Virtanen JK, Mursu J, Voutilainen S, Tuomainen TP. Serum long-chain $n-3$ polyunsaturated fatty acids and risk of hospital diagnosis of atrial fibrillation in men. Circulation 2009; 120: 2315-2321. 